\title{
Obituary news May-17
}

BMA would like to express deep condolence on deaths of the following notable physicians in recent past:

\begin{tabular}{|c|l|c|l|c|}
\hline SL.No & Name & Age & Name of District & Date of Death \\
\hline 1 & Dr. Md. Babor Ali & 52 & Naogaon & $14 / 10 / 2016$ \\
\hline 2 & Dr. Suman Shikder & - & Magura & $13 / 1 / 2017$ \\
\hline 3 & Dr. K.M. Ashraf-uz-Zaman & & Rajshahi & $15 / 1 / 2017$ \\
\hline 4 & Dr. Romesh Chandra Sarkar & - & Khulna & $22 / 01 / 2017$ \\
\hline 5 & Dr. Shafique Ahmed Khan & 51 & Dhaka & $23 / 01 / 2017$ \\
\hline 6 & Dr. Md. Iftekhar Kashem & 53 & Dhaka & $24 / 01 / 2017$ \\
\hline 7 & Dr. Md. Motarrab Hossain & 85 & Bogra & $27 / 01 / 2017$ \\
\hline 8 & Dr. S.M,A. Latif & 92 & Bagerhat & $24 / 02 / 2017$ \\
\hline 9 & Mr. Suranzit Sen Gupta & 72 & Ex-Minister & $05 / 2 / 1017$ \\
\hline 10 & Dr. Jaynal Abedin & - & Rajshahi. & $12 / 03 / 2017$ \\
\hline 11 & Language Fighter Dr. Ali Ajgar & 87 & & $31 / 03 / 2017$ \\
\hline 12 & Dr. Anowar Hossain & - & & \\
\hline 13 & Dr. Khurshed Alam & 35 & Dhaka & $25 / 04 / 2017$ \\
\hline 14 & Dr. Abdul Mannan Sarker & 41 & Belkuchu, Sirajgonj & $05 / 05 / 2017$ \\
\hline 15 & Dr. Kamol Krishna Karmakar & - & Barisal & \\
\hline 16 & Dr. Md. Nasir Uddin & 75 & Professor, Dept. of Anatomy,Myneshingh Medical College & \\
\hline 17 & Dr. M.A. Quader & 81 & Ex.Pricipal, Rajshahi Medical College & $27 / 5 / 2017$ \\
\hline 18 & Dr. A.N.M.Foqruz-Zaman & 50 & Sinior Lecturer, Chattagong Medical College & \\
\hline 19 & Dr. Margaret Munghereta & - & (past President-2013-2014, World Medical Association & \\
\hline 20 & Mrs. Jahanara Jaman & 84 & Ex-Home Minister & \\
\hline
\end{tabular}

May Allah bless the departed souls. Our heartiest commiseration to the deceased's family, our prayers are with them during this difficult moment of their life. 